ARTICLE

\title{
Memory biases in alcohol use disorder: enhanced memory for contexts associated with alcohol prospectively predicts alcohol
}

\section{use outcomes}

\author{
Elizabeth V. Goldfarb ${ }^{1,2}$, Nia Fogelman ${ }^{1,2}$ and Rajita Sinha ${ }^{1,2,3}$
}

\begin{abstract}
Memory for prior drinking experiences may powerfully drive later alcohol use in familiar drinking contexts, yet we know little about what patients with alcohol use disorder (AUD) remember of alcohol-related episodes. Although animal and theoretical models of addiction emphasize the importance of different memory systems for understanding maladaptive use, clinical research parsing what AUD patients remember from alcohol-related episodes is lacking. The current study applied a novel memory task in which moderate drinkers $(N=30)$ and treatment-seeking individuals with alcohol use disorder (AUD: $N=29)$ encoded associations between photographs of objects (alcoholic beverages and neutral items) and photographs of neutral scenes. At least $24 \mathrm{~h}$ later, two types of memory were assessed: item memory (object recognition) and associative memory (cued recognition of scenes associated with objects). To assess which memories predicted drinking, real-world behavior was assessed in patients with AUD at baseline and for 4 weeks following memory tests. Despite demographic differences, the results showed broadly impaired item memory in AUD compared with moderate drinkers $(p<0.001)$, but enhanced associative memory for scenes paired with alcohol $(p=0.015)$. These associative memory biases were especially pronounced for stimuli rated as more affectively salient. Furthermore, stronger but less detailed memory for alcohol-related associations (i.e., choosing the correct scene but the incorrect photograph) significantly predicted heavier baseline $(p=0.002)$ and higher subsequent $(p=0.01)$ drinking in patients with AUD. These findings reveal a novel alcohol-related memory bias in AUD, and uncover the importance of associative memory for understanding real-world heavy alcohol use.
\end{abstract}

Neuropsychopharmacology (2020) 45:1297-1305; https://doi.org/10.1038/s41386-020-0650-y

\section{INTRODUCTION}

Alcohol use disorder (AUD) is a significant public health problem, with recent estimates that 14.5 million people in the United States meet diagnostic criteria [1]. This disorder is also difficult to treat, as patients with AUD often relapse in the presence of alcohol-related environmental cues [2, 3]. These cues influence behavior by triggering retrieval of memories for past drinking episodes $[4,5]$. Across species, learning and memory are thought to play a crucial role in addiction [5-9]. However, we know very little about what patients with AUD actually remember about alcohol-related episodes. Understanding how alcohol-related memories are formed and guide maladaptive drinking behavior may be critical for developing effective behavioral and pharmacological treatments [10], and translating findings from animal models of alcohol addiction. Here, we aimed to uncover novel biases in different types of memories for alcohol-related episodes in AUD, and identify memories that prospectively predict drinking behavior.

Decades of research across species have demonstrated that we can form different types of memories, which may each contribute to drinking $[2,11,12]$. These different types of memories can be formed from a single event [11, 13]. Following recent suggestions that memory for a single event (episodic memories) may drive problematic substance use [9], there is a need to understand which parts of episodic memories contribute to drinking. Episodic memories can be parsed into representations of items, or individual parts of an event, and associations, in which relationships between different elements are integrated [14]. Neuroimaging and lesion studies demonstrate that these representations are supported by distinct neural systems [15-17] and (as discussed below), are differentially sensitive to emotional content. The relative strength of these different memories may be clinically meaningful. For example, after a traumatic event, having impaired memory for associations, but strong memory for items, may contribute to symptoms of post-traumatic stress disorder (like responding to trauma-related items in nonthreatening contexts) $[18,19]$. However, we know very little about the significance of these different types of memory in addictive disorders like AUD.

To date, clinical research on alcohol-related memories in AUD has been limited to short-term assessments of item memory [20-22]. Similarly, current therapeutic approaches focus on modifying item memories $[23,24]$. Thus, whether patients with AUD have biased memory for alcohol-related associations, and whether either item or associative memory can predict problematic drinking behavior, remains unknown. Understanding which types of alcohol-related memories characterize AUD and heavy alcohol use could be of use in designing targeted behavioral interventions [25].

\footnotetext{
${ }^{1}$ Yale Stress Center, Yale School of Medicine, New Haven, CT, USA; ${ }^{2}$ Department of Psychiatry, Yale School of Medicine, New Haven, CT, USA and ${ }^{3}$ Department of Neuroscience, Yale School of Medicine, New Haven, CT, USA

Correspondence: Rajita Sinha (rajita.sinha@yale.edu)
}

Received: 26 November 2019 Revised: 19 February 2020 Accepted: 25 February 2020

Published online: 3 March 2020 
There is compelling evidence that alcohol-related items will be remembered differently from associated neutral contexts. For example, memory for affectively salient items (e.g., an injured person) is typically enhanced, while memory for neutral information associated with these items (e.g., a background scene) is impaired [26-28]. Alcohol-related items are highly affectively salient for heavy drinkers [29] and patients with AUD [30], and limited evidence indicates that these patients have better memory for alcohol-related items after a short delay [20, 21] (but see ref. [22]). This suggests that patients with AUD would have improved longterm memory for alcohol-related items at the expense of associations. However, patients with AUD may break away from this typical emotional memory bias. Compared with healthy controls, patients with AUD showed stronger approach behavior based on alcohol-related associations [31], and rodents chronically exposed to ethanol were better at learning ethanol-related associations [32,33]. Similarly, drinking alcohol before encoding alcohol-related information improved associative (but not item) memory [34], and acute stress before encoding salient information led to enhanced associative (but not item) memory [35]. As AUD is associated with elevated basal stress hormones [36] and hippocampal volume changes [37], we hypothesized that chronic alcohol intake in AUD could result in an unusual memory bias: enhanced memory for alcohol-related associations, but not items [12].

Memory for alcohol-related associations may also be especially important for predicting later drinking behavior [38-40]. Neutral contexts associated with alcohol are a powerful trigger for relapse across species [2], and neutral stimuli known to predict drug reinforcement can themselves promote drug-seeking behavior $[41,42]$. Recent work on memory-guided decision-making in humans emphasizes the importance of associative memories for driving later behavior $[9,43,44]$. However, these associative memories may not be very precise. Patients with AUD have been shown to overgeneralize autobiographical memories [45], a process associated with responding inappropriately to cues or contexts that are vaguely similar to one's past experiences (for discussion in the fear domain, see ref. [46]). Thus, it is crucial to measure not only what patients remember about alcohol-related events, but also how detailed these memories are.

Here, we aimed to characterize long-term memory for alcoholrelated episodes in AUD, and determine which memories predict future drinking. We measured memory for individual objects (item memory) and scenes paired with these objects (associative memory), distinguishing how precisely individuals remembered these associations. Translating findings from the basic and clinical literature, we hypothesized that patients with AUD would show worse memory for items, but a bias toward enhanced memory for alcohol-related associations compared with moderate drinkers. We further hypothesized that this greater bias toward remembering alcohol-related associations would predict future drinking in AUD.

\section{MATERIALS AND METHODS}

Participants

Fifty-nine individuals between the ages of 18 and 57 participated in the study. Both participant groups were recruited from the community via separately worded advertisements in local newspapers, web-based advertisements, and flyers posted for research participation. Moderate drinkers (MOD) were asked if they "liked beer" or wanted to participate in research; treatment-seeking patients with AUD were recruited with advertisements asking if they "wanted alcohol treatment" or "wanted to cut back on drinking", and were only enrolled if they endorsed wanting to cut back or abstain during an initial phone screening. Intake assessments measured demographics and past drinking behavior, followed by encoding and memory sessions of the item and associative memory task (described below). Participants were screened to ensure that they did not meet current criteria for substance use disorder for another psychoactive substance (excluding nicotine), have significant underlying medical conditions, or regularly use any of the following medications: anticonvulsants, sedatives/hypnotics, prescription analgesics, other antihypertensives, anti-arrhythmic, antiretroviral medications, tricyclic antidepressants, SSRIs, naltrexone, or antabuse. Participants were also excluded if they were intoxicated at any laboratory visit (breathalyzer values $>0$ ).

Participants belonged to one of two groups: MOD and treatment-seeking patients with AUD. MOD were defined as individuals who had a total AUDIT score less than 7, never met DSM-V criteria for AUD, were below weekly hazardous drinking levels (defined by the National Institute on Alcohol Abuse and Alcoholism criteria as $7+$ drinks/week for women and $14+$ drinks/ week for men), and rarely ( $\leq 6 \times /$ year) drank at binge levels (4+ drinks/occasion for women and $5+$ drinks/occasion for men). AUD patients were treatment-seeking individuals who met current DSM-V criteria for AUD on the Structured Clinical Interview for the DSM-V [47]. An additional six participants were excluded. ${ }^{1}$ Participants provided written informed consent to complete the study, and all procedures were approved by the Yale Medical School Institutional Review Board.

\section{Intake assessments}

To determine current drinking severity in both groups, drinking behavior was assessed at intake using the Alcohol Use Disorders Identification Test [48]. All participants were also evaluated using the Structured Clinical Interview for DSM-V to determine current AUD diagnoses (AUD group) or no past or current AUD or other substance use disorder diagnoses (MOD group) [47]. The Shipley Institute of Living Scale was used to measure IQ [49].

\section{Procedures}

Following intake assessments, all participants were scheduled for two separate sessions of encoding (Day 1) and retrieval (Day 2), described below (Fig. 1a). These sessions occurred at least $24 \mathrm{~h}$ apart (mean $=3.95$ days), and each lasted for 30-45 min, and participants were alcohol and drug-free for each as per breathalyzer and urine toxicology screens. AUD patients were in the early phase (i.e., tested within the first month) of weekly outpatient behavioral counseling treatment for AUD, using the standardized 12-step counseling treatment manual [50], provided by a licensed alcohol/drug abuse counselor.

Item and associative memory task

Encoding. The design of the task is similar to the one recently used to assess the influence of acute stress on item and associative memory [35] (Fig. 1b). On Day 1 (encoding), participants were shown 40 unique pairs of objects and scenes ( $5 \mathrm{~s}$ ), and instructed to vividly imagine the object as part of the scene. These 40 object/ scene pairs were split into two lists (order counterbalanced). In one list $(N=20)$, objects were photographs of alcoholic beverages on a white background. In the other list $(N=20)$, objects were photographs of common neutral items on a white background (as in ref. [51]; see Fig. 1c for examples). This design thus provided a baseline assessment of non-alcohol-related memory functioning

\footnotetext{
${ }^{1}$ Three participants were excluded due to technical issues (MATLAB crashing: $N=1$ MOD; pressing wrong keys: $N=1$ AUD) and comprehension issues (thought memory was being assessed for information presented that same day; $N=1 \mathrm{MOD}$ ). Three additional participants were excluded based on a priori criteria for very poor memory performance (hits $<$ misses and $d^{\prime}<0$; [35]; $N=1 \mathrm{MOD}, N=2$ AUD). Memory was computed based on mean performance pooled across alcohol and neutral object cues in order to avoid biases due to either cue type.
} 
A

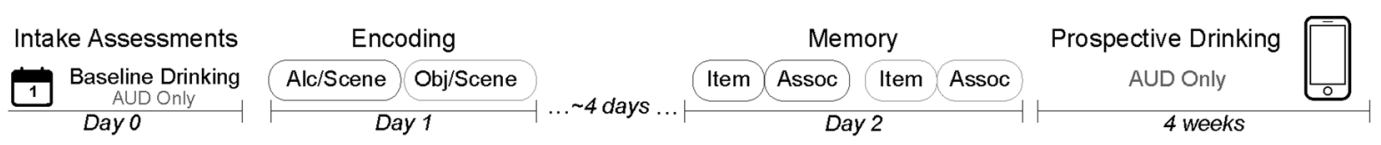

B
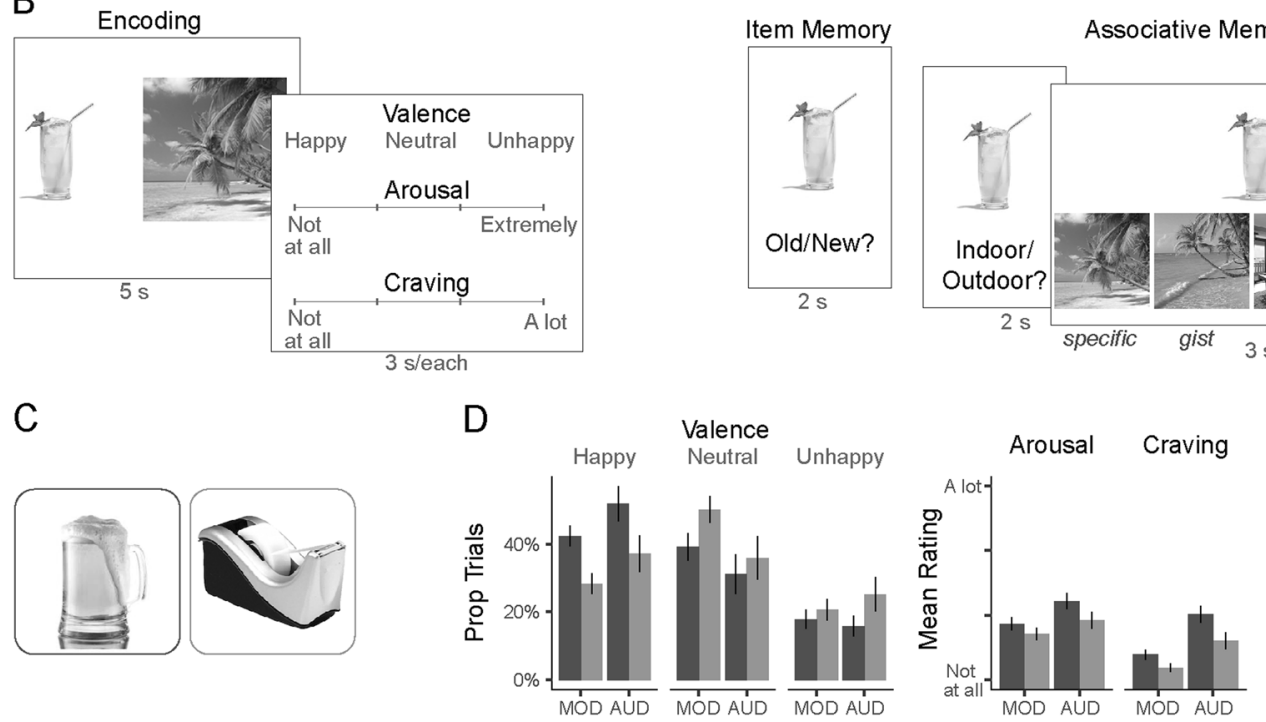

Alcohol

Neutral Object

Fig. 1 Design and subjective responses during encoding. a Procedure. Participants first completed intake assessments, in which baseline real-world drinking behavior (over the previous week) was assessed in patients with alcohol use disorder (AUD). Next, participants completed two blocks of object/scene encoding with alcoholic beverages or neutral objects. Cue order (alcohol vs. neutral objects) was counterbalanced across participants. After a delay, they were tested for their memory for the individual items and their associated scenes. Finally, patients with AUD were tracked for 4 weeks to assess prospective real-world drinking behavior. b Task design. During encoding, participants vividly associated unique pairs of objects and scenes. Ratings of subjective responses were assessed for every trial. During the item memory test, recognition of individual objects (plus content-matched foils) was assessed. During the associative memory test, participants were asked to recall whether the scene paired with the object was indoor or outdoor, and then to select the scene from a list of photographs. These photographs contained the exact image seen during encoding (specific associative memory), and an image portraying the same type of scene, but a different photograph (gist associative memory). c Examples of alcohol-related objects (red border, left) and neutral objects (gray border, right). d Subjective ratings during encoding for object/scene pairs containing alcohol (red) or neutral objects (gray). MOD moderate drinkers, AUD patients with alcohol use disorder. Error bars $=+/-1 \mathrm{SE}$.

per participant. Scene photographs were taken from a standardized database, and did not show any people [52]. An equal number of indoor and outdoor scenes were presented per list. To facilitate memory performance, participants were instructed that their memory for object/scene pairs would be tested, and were encouraged to imagine stories about the object interacting as part of the scene.

After vividly imagining each object/scene pair, participants next used the keyboard to rate their subjective responses (3 $\mathrm{s}$ each): how they felt when imagining the object/scene pair (happy/ unhappy/neutral); how intensely they felt that way (4-point scale: $1=$ not at all intense; $4=$ extremely intense); how much they wanted an alcoholic drink ( $1=$ not at all; $4=$ a lot). Responses were illuminated on the screen in green, with trials separated by intertrial intervals (ITI, $1 \mathrm{~s}$ ).

Memory. On Day 2, participants returned to the laboratory for memory assessments. Lists were presented in the same order as encoding.

To assess item memory, participants were shown the objects studied at encoding $(N=40)$ intermixed with novel foils congruent with each list ( $N=40 ; 3 \mathrm{~s}$ each). For example, images of novel alcoholic beverages were intermixed with images of previously studied alcoholic beverages. On each trial, participants reported whether each object was old (previously studied) or new (never studied) on a 4-point scale (confident old; not sure old; not sure new; confident new; $2 \mathrm{~s}$ per response with $1 \mathrm{~s}$ ITI).

Next, we conducted a two-part assessment of associative memory. First, participants viewed each of the objects studied at encoding ( $3 \mathrm{~s})$, and were asked to indicate whether this object had been paired with an indoor or outdoor scene (recall, $2 \mathrm{~s}$ ). Next, they were shown the object along with four scene photographs. The scenes included the exact photograph presented during encoding, a different scene that was presented at encoding (to control for familiarity), and two matched perceptual/schematic lures portraying the same scenes. Participants were instructed to select the scene that was presented with the object during encoding (recognition, $3 \mathrm{~s}$ ). Participants were further told that, if they remembered what scene was paired with the object (in Fig. 1, a beach), but not exactly which photograph was used, to make their best guess between the two photographs portraying that scene (i.e., either of the two beach photographs). Selection of the correct scene and correct picture indicated "specific" associative memory (i.e., detailed memory for the paired scene), whereas selection of the correct scene but incorrect picture indicated "gist" associative memory (i.e., less precise memory, with participants being fooled by the similar scene).

Real-world drinking behavior

Alcohol use in patients with AUD $(N=27)$ was assessed at baseline (upon enrollment in the study) using the Substance Use Calendar [53] based on the time-line follow-back method [54]. Prospective drinking was measured using daily diary-based assessment via smartphone-based prompts for 4 weeks as described previously [55]. Every evening (8:00 p.m.), participants were asked whether they consumed wine, beer, or liquor that day, and if so, to indicate the number of drinks they consumed. Participants were given a 6-h window each day to respond, and compliance was monitored 
throughout the study. Participants were paid \$2 per day for responding to surveys, and received an additional $\$ 10$ bonus for completing all surveys that week. On average, participants completed $74.3 \%$ of prompts over 4 weeks. One participant completed only one $(<5 \%)$ smartphone prompt and was excluded from analyses. The average number of drinks per day were computed for each of the 4 weeks. Days on which data were not collected (e.g., participants failed to answer the prompt) were dropped from the calculations.

\section{Analytic approach}

The goal of these analyses was to characterize ways in which alcohol-related memories differed between patients with AUD and MOD, and to determine which alcohol-related memories best predicted later real-world drinking behavior. All analyses were performed in $\mathrm{R}$ (ref. [56], 3.4.1).

To accomplish the first goal, we used linear mixed effect (LME) models to describe memory as a function of Group (MOD vs. AUD) and Cue (alcohol vs. neutral object) with a random intercept and additional between-subject covariates (nlme package [57]). These covariates included participant features of age and IQ (as the groups differed significantly in these dimensions, Table 1), and experiment features of cue order (i.e., whether neutral objects or alcoholic beverages were shown first), and the number of days between encoding and retrieval. All reported the main effects of Group, as well as Group $\times$ Cue interactions were consistent even when these covariates were not included in the model. To further establish that these effects were driven by the severity of alcohol dependence, all models with main effects of Group or Group $\times$ Cue interactions were repeated, focusing on patients who met the criteria for severe AUD. Effect sizes $\left(\eta^{2}\right)$ were computed using the sjstats package for mixed models (following [58]), and interpreted as low, moderate, and large effects relative to the established benchmarks [59].

Item-level memory was quantified using $\mathrm{d}^{\prime}$, or the normalized ratio of hits (proportion of previously viewed objects correctly identified as old) to false alarms (FA, proportion of new objects incorrectly identified as old). If significant effects on $d^{\prime}$ were observed, further analyses considered hits and FA separately.

Associative memory was quantified using performance on cued recall and recognition. To probe the specificity of these memories, we created a combined metric to limit the possibility of random guesses during recognition, and focus on trials that were robustly remembered. Of the trials in which participants correctly recalled the scene category (indoor vs. outdoor), specific memory was computed as the proportion of trials from which the exact scene photograph was selected, and gist memory as the proportion of trials for which the correct scene, but the

Table 1. Participant demographics.

\begin{tabular}{llll}
\hline & $\begin{array}{l}\text { Moderate } \\
\text { drinkers (MOD) } \\
N=30\end{array}$ & $\begin{array}{l}\text { Alcohol use } \\
\text { disorder (AUD) } \\
N=29\end{array}$ & Difference? \\
\hline Gender (\% female) & $50 \%$ & $48.2 \%$ & $p>0.25$ \\
Age (mean [SD]) & $27.23[8.23]$ & $35.86[11.22]$ & $p=0.001$ \\
IQ & $113.07[6.37]$ & $102.9[10.51]$ & $p<0.001$ \\
Current smoker & $6.7 \%$ & $58.6 \%$ & $p<0.001$ \\
AUDIT: total & $2.57[1.76]$ & $20.86[6.86]$ & $p<0.001$ \\
$\begin{array}{l}\text { Average drinks/ } \\
\text { week }\end{array}$ & $2.08[2.56]$ & $38.41[51.4]$ & $p<0.001$ \\
$\begin{array}{l}\text { Max drinks/ } \\
\text { episode }\end{array}$ & $3.17[1.9]$ & $12.14[12.45]$ & $p<0.001$ \\
\hline Age and IQ were included as covariates in all analyses. & \\
\hline
\end{tabular}

wrong photograph was selected. Additional analyses in which specific and gist memory were computed out of all possible trials are presented in Fig. S1.

To test whether memory differed as a function of emotional responses at encoding, we used LME models to describe memory on all trials as a function of Group (MOD vs. AUD) and Cue Ranking (described below; top-ranked alcohol, other alcohol, and neutral objects) with the same covariates described above.

Prospective prediction of drinking over the 4-week period following the memory task was assessed using LME with a random intercept. Item and associative (specific and gist) memory were then assessed in three separate models to predict drinking. In each model, both alcohol- and neutral object-related memory were included as predictors to account for baseline differences in memory performance.

\section{RESULTS}

Participant demographics

Thirty MOD and 29 patients with AUD were included in the final sample (Table 1). On average, patients with AUD had a total AUDIT score over 20, tended to drink >38 drinks each week, and consumed a maximum of 12 drinks per drinking episode. They endorsed an average of 6.45 symptoms on the SCID (range: 2-10), and $58.6 \%$ met criteria for severe AUD. On average, they had not had a drink in about 5 days at the time of encoding (range: 0-37 days), and this measure was not related to memory performance.

In contrast, MOD had an average total AUDIT score under three, drank approximately two drinks per week, and consumed a maximum of about three drinks per episode. Groups did not differ in gender, but did differ in age and IQ, leading these to be included as covariates in all analyses (see "Analytic approach"). Although smoking status differed significantly between moderate drinkers and patients with AUD, smoking status was not predictive of memory performance (see Table S2 for effects of these covariates in all models).

\section{Affective responses during encoding}

Imagining alcoholic beverages in neutral scenes induced strong affective responses across our sample (Fig. 1b). scene pairs/scene pairs, participants rated significantly more alcohol/scene pairs as making them feel "happy" $\left(F_{1,57}=26.31, p<0.001, \eta^{2}=0.245\right)$. These trials were also rated as significantly more arousing (main effect of Cue [alcohol vs. neutral object]: $F_{1,57}=11.92, p=0.001$, $\left.\eta^{2}=0.132\right)$ and inducing significantly higher craving $\left(F_{1,57}=32.74\right.$, $\left.p<0.001, \eta^{2}=0.244\right)$. Overall, patients with AUD provided significantly higher ratings of both arousal and craving (arousal, main effect Group: $F_{1,54}=4.05, p=0.049, \eta^{2}=0.045$; Craving: $\left.F_{1,54}=16.76, p<0.001, \eta^{2}=0.125\right)$. Alcohol/scene pairs induced numerically stronger craving in patients with AUD (Cue $\times$ Group: $\left.F_{1,57}=3.81, p=0.056, \eta^{2}=0.028\right)$, an effect that was statistically significant when focusing on patients with severe AUD (Cue $\times$ Group: $F_{1,45}=4.85, p=0.03, \eta^{2}=0.042$ )

Item memory differences

Overall, patients with AUD had worse item memory than MOD ( $d$ ': main effect Group, $F_{1,53}=21.72, p<0.001, \eta^{2}=0.139$, Fig. 2a) with significantly more FA $\left(F_{1,53}=7.16, p=0.01, \eta^{2}=0.051\right)$. Both groups had higher item memory for neutral objects than alcoholic beverages (d': main effect Cue: $F_{1,57}=16.12, p<0.001, \eta^{2}=0.103$ ), driven by higher $F A$ for alcohol $\left(F_{1,57}=17.43, p<0.001, \eta^{2}=0.125\right.$; no significant cue differences in hit rate; $p>0.25$ ). This pattern did not differ significantly between groups (Group $\times$ Cue, $d^{\prime}: F_{1,57}=$ $2.37, p=0.13$; FA: $F_{1,57}=1.63, p=0.21$; memory by cue and group shown in Table S1). These effects remained significant when restricting analyses to compare MOD to patients with severe AUD 
A Item Memory

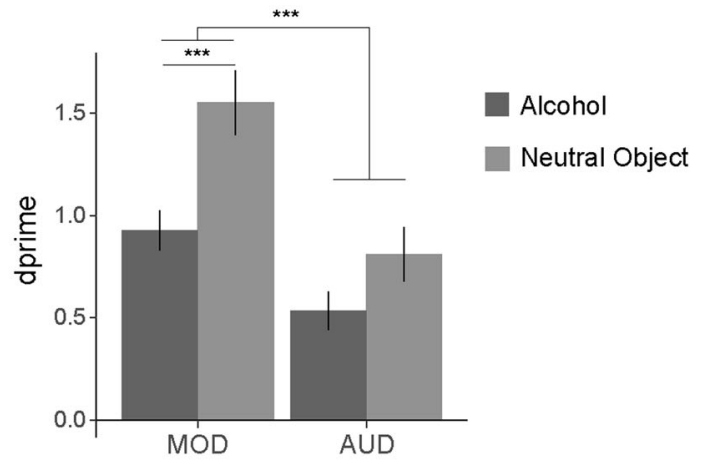

B Associative Memory

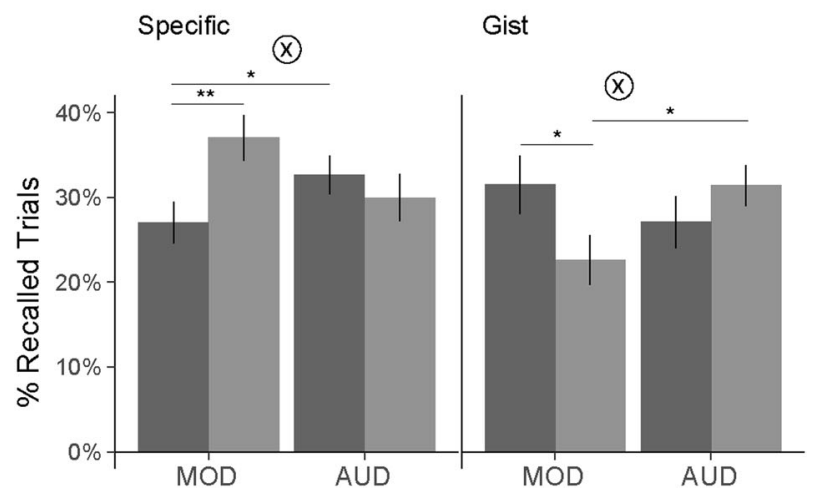

Fig. 2 Drinking history influences item and associative memory for alcohol cues. a Patients with alcohol use disorder (AUD) had significantly worse item memory than moderate drinkers (MOD). b Distinct effects of alcohol cues on associative memory in moderate drinkers and patients with AUD. Left, specific (detailed) memory for alcohol-related associations is impaired in moderate drinkers, but spared in patients with AUD. Right, moderate drinkers show less detailed "gist"-level memory for alcohol-related associations, whereas patients with AUD show less detailed memory for associations with neutral objects. ${ }^{*} p<0.05 ;{ }^{* *} p<0.01 ;{ }^{* * *} p<0.001$.

( $d^{\prime}$, main effect Group, $F_{1,45}=15.7, p<0.001, \eta^{2}=0.124$; main effect Cue: $F_{1,45}=24.65, p<0.001, \eta^{2}=0.194$ ).

Associative memory differences

Cued recall. We did not observe significant differences in cued recall (choosing whether the item was paired with an indoor or outdoor scene) as a function of group $\left(F_{1,53}=0.01, p>0.25\right)$, cue type $\left(F_{1,57}=0.71, p>0.25\right)$, or their interaction $\left(F_{1,57}=0.74, p>\right.$ 0.25 ), which may have been due to the difficulty of this task (performance summarized in Table S1). There were also no significant differences in cued recall when focusing on patients with severe AUD (all $p>0.25$ ).

Specific recognition. Unlike item memory, patients with AUD did not have significantly worse associative memory than MOD (main effect Group: $p>0.25$ ). Associative memory also did not differ significantly between trials containing alcohol and neutral objects (main effect Cue: $F_{1.57}=2.15, p=0.15$ ). However, we did observe a significant interaction, demonstrating distinct biases in memory for alcohol-related associations as a function of drinking group (Group $\times$ Cue, $F_{1,57}=6.3, \quad p=0.015, \eta^{2}=0.052$, Fig. $2 b$, left; Table S1).

In MOD, memory for scenes associated with alcohol was significantly lower compared with scenes associated with neutral objects $(b=-0.1$ [SE $=0.04], p=0.007)$. In contrast, patients with AUD did not show this deficit $(b=0.03$ [0.04], $p>0.25)$. In fact, relative to MOD, patients with AUD showed significantly better memory for scenes associated with alcohol $(b=0.09$ [0.04], $p=$ $0.027)$. These group differences became stronger when focusing on patients with severe AUD (Group $\times$ Cue: $F_{1,45}=10.32, p=$ $0.002, \eta^{2}=0.096$; alcohol-paired scenes only: Group $b=0.14$ [0.05], $p=0.006$ ). The same pattern of results held when analyzing responses on all trials (not only those with correct cued recall, Fig. S1).

A similar pattern was observed when comparing memory performance to chance (25\%). In MOD, memory for scenes paired with neutral objects was significantly above chance $\left(37 \% ; t_{29}=\right.$ 4.56, $p<0.001)$, but memory for scenes paired with alcohol was not $(27 \% ; p>0.25)$. In contrast, patients with AUD had abovechance memory for scenes paired with alcohol $\left(32.7 \% ; t_{28}=3.43\right.$, $p=0.002)$, with memory for scenes paired with neutral objects differing at a trend level (29.9\%; $\left.t_{28}=1.78, p=0.085\right)$. Together, these results demonstrate spared, and in fact, enhanced memory for detailed alcohol-related associations in AUD.

Gist recognition. Having shown that patients with AUD and MOD differed in their ability to remember detailed associations with alcohol-related cues, we next examined less detailed, "gist"-level associative memories. Although there were no overall differences between groups $(p>0.25)$, or between alcohol and neutral object cues $(p>0.25)$, we did observe a significant Group $\times$ Cue interaction $\left(F_{1,57}=5.1, p=0.028, \eta^{2}=0.041\right.$, Fig. $2 b$, right). Moderate drinkers had significantly more "gist"-level memories for associations with alcohol than neutral objects $(b=0.09$ [0.04], $p=$ $0.034)$, but patients with AUD did not show this bias $(p>0.25)$. Instead, compared with MOD, patients with AUD had significantly more "gist"-level memories for associations with neutral objects $(b=0.12$ [0.05], $p=0.014)$. As with specific recognition, these group differences became stronger when focusing on patients with severe AUD (Group $\times$ Cue: $F_{1,45}=6.55, p=0.014, \eta^{2}=0.066$; neutral object-paired scenes only: Group $b=0.14$ [0.06], $p=$ 0.015).

Together, these results demonstrate a novel associative memory bias in AUD. MOD had weaker and less precise memories for associations with alcohol. However, patients with AUD had relatively stronger and more precise memory for alcohol-related associations, even when their memory for associations with neutral objects was impaired.

Relationship between affective responses and memory One mechanism that could drive differences in memory for alcohol-related information involves affective responses. Although alcohol-related trials were overall rated as inducing more arousal and craving, we wanted to test whether variability in these subjective responses influenced group-level differences in alcoholrelated memories (Fig. 3a).

Consistent with prior findings, we found that affective salience enhanced item memory across groups (Fig. 3b; hit rate: main effect of Cue Ranking [top-ranked alcohol, other alcohol, and neutral objects], $F_{2,104}=4.67, p=0.011, \eta^{2}=0.067$, Fig. $3 b$ ). This effect did not differ between groups (Cue Ranking $\times$ Group: $p>0.25$ ).

However, although affective salience often impairs memory for associations, this was not the case for both drinking groups (Fig. 3c, Cue Ranking $\times$ Group: $F_{2,104}=3.96, p=0.02, \eta^{2}=0.05$ ). MOD indeed had worse specific associative memory for affectively salient pairs (top-ranked alcohol vs. neutral objects: $b=-0.1$ [0.04], $p=0.011)$. In contrast, patients with AUD did not show this impairment $(b=0.05[0.04], p=0.19)$. Instead, patients with AUD had significantly better memory for affectively salient pairs compared with MOD ( $b=0.12$ [0.04], $p=0.007)$. There were no significant group differences in the effects of affective salience on 


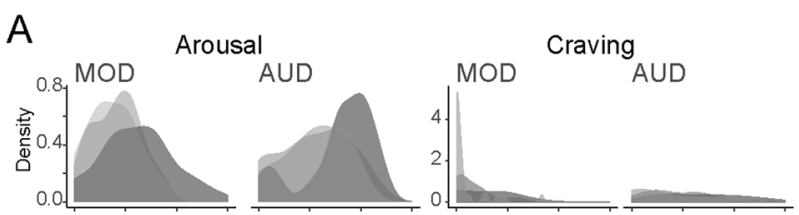

Top-Ranked Alcohol Other Alcohol Neutral Object
B Item Memory: Hits

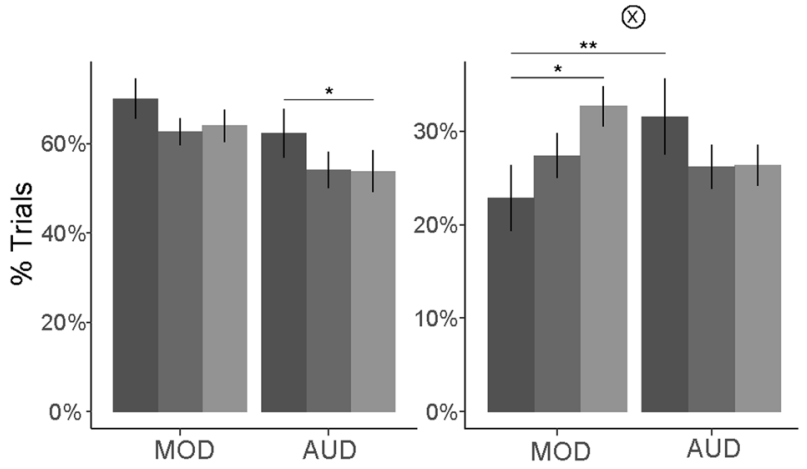

Fig. 3 Affective responses to alcohol drive memory biases. a Density plots of arousal and craving ratings for trials containing photographs of the top-ranked alcoholic beverage. We computed affective responses per alcoholic beverage (weighted sum of arousal and craving with penalty for rating trials as neutral), and separated trials including the highest-ranked alcoholic beverage ("top-ranked alcohol") from other alcohol and neutral object trials. b Item memory separated by drinking group and alcohol ratings, showing better item memory for more salient alcohol items. c Associative memory (specific) separated by drinking group and alcohol ratings. More salient alcohol items led to worse associative memory in moderate drinkers (MOD), but better associative memory in patients with alcohol use disorder (AUD). Gray bars (neutral objects) reproduced from Fig. $2 c$. Error bars $= \pm 1$ SE. ${ }^{*} p<0.05,{ }^{* *} p<0.01$.

"gist"-level associative memory (Cue Ranking $\times$ Group: $F_{2,104}=$ $1.62, p=0.2$ ).

These results suggest that affective salience had opposite effects on memory for items and associations in MOD, but congruent effects across memories in AUD. We tested this directly by analyzing performance as a function of Memory Type (hits vs. specific associative memory) and Cue Ranking. Among MOD, we found a significant interaction between salience and memory type (Cue Ranking $\times$ Memory Type: $F_{2,135}=4.26, p=0.016, \eta^{2}=0.024$ ), demonstrating that affective salience had opposite effects on item and associative memory. In contrast, patients with AUD did not show this interaction $(p>0.25)$. Instead, patients with AUD showed stronger memory for more affectively salient associations, although this was not statistically significant (main effect Cue Ranking: $F_{2,125}=2.74, p=0.068, \eta^{2}=0.022$ ). These results demonstrate that emotional responses to alcohol-related information broadly amplified memory in patients with AUD, while having uneven effects on memory in MOD.

Predicting drinking with memory

Having shown distinct biases in memory for alcohol-related information in AUD, we next conducted exploratory analyses to test whether alcohol-related memories predicted real-world drinking behavior among patients with AUD (Figs. 4 and S2). We used alcohol-related item and associative memory as predictors of drinking (average drinks per day) in AUD participants. To account for baseline differences in memory, we controlled for neutral object-related memories in all analyses.
Baseline drinking behavior. We first tested whether memory biases were associated with differences in baseline alcohol consumption (Fig. 4a). Although item-level memory differed between groups, there was no significant relationship between memory for alcohol-related items and baseline drinking behavior $\left(d^{\prime}, p>0.25\right)$. In contrast, having stronger "gist"-level memory for scenes associated with alcohol (but not specific memory, $p>0.25$ ) predicted higher daily levels of drinking at baseline $\left(F_{1,22}=11.68\right.$, $\left.p=0.002, \eta^{2}=0.345\right)$. This relationship persisted when drinking severity (total AUDIT scores) was included in the model (gist memory: $F_{1,21}=13.44, p=0.001, \eta^{2}=0.285$; total AUDIT: $F_{1,21}=$ $\left.12.51, p=0.002, \eta^{2}=0.266\right)$.

Prospective drinking behavior. We next tested whether memory biases had prospective predictive validity by predicting drinking over the course of 4 weeks following the memory assessments (Fig. 4b). Notably, as prospective drinking was measured during treatment, this metric of drinking was not correlated with baseline drinking behavior $(p>0.25)$, providing an opportunity for replicating the relationship between memory and drinking with $a$ separate metric.

Consistent with baseline drinking, there was no significant effect of alcohol-related memory for items ( $d^{\prime}: p>0.25 ; d^{\prime} \times$ Week: $p>0.25$ ) or specific associations (Specific: $p>0.25$; Specific $x$ Week: $p>0.2$ ) on subsequent daily drinking. Strikingly, we found that "gist"-level memory for alcohol-related associations predicted not only baseline but also subsequent daily levels of drinking $\left(F_{1,23}=7.87, p=0.01, \eta^{2}=0.082\right)$. The same pattern of results held when using "gist"-level memory performance on all trials (and not only those with correct cued recall; Fig. S1).

\section{DISCUSSION}

The current study revealed novel and meaningful biases in alcoholrelated memories among patients with AUD. Although patients had impaired memory for items and neutral associations relative to moderate drinkers, their memory for alcohol-related associations was relatively spared. In exploratory analyses, stronger, but less detailed, "gist" memory for alcohol-related associations predicted both baseline and prospective drinking during the next 4 weeks. These findings highlight the importance of assessing different memory processes in AUD, and the relevance of memory biases for alcohol use outcomes.

Consistent with previous reports of deficits in neutral episodic memory [60-63], we found that patients with AUD had impaired memory for non-alcohol-related items. However, by also measuring memory for alcohol and non-alcohol-related specific and gist associations, the current study revealed novel biases toward enhanced memory for alcohol-related associations. This finding aligns with theories that memories for drug-related information are prioritized in addiction [5], and may have consequences for later alcohol-related decisions. A recent body of work has demonstrated the role of associative memory in guiding decision-making and choices (e.g., refs. [43, 44, 64, 65]). Together, these previous and current findings are consistent with a cognitive mechanism by which biases toward remembering alcohol-related associations would tip decisions toward selecting alcohol-related options, and support recent proposals for developing therapeutic interventions that bolster memory for non-drug-related associations [9].

The current design provided the opportunity to differentiate aspects of memory that distinguished recreational drinkers from patients with AUD (perhaps due to disease-related neuroadaptations) from those that predicted current disease symptoms (increased drinking). We found that item memory differed between groups, but did not explain drinking levels. In contrast, memory for scenes associated with alcohol both distinguished patients with AUD from moderate drinkers (medium-effect size) and predicted 
A Baseline drinking
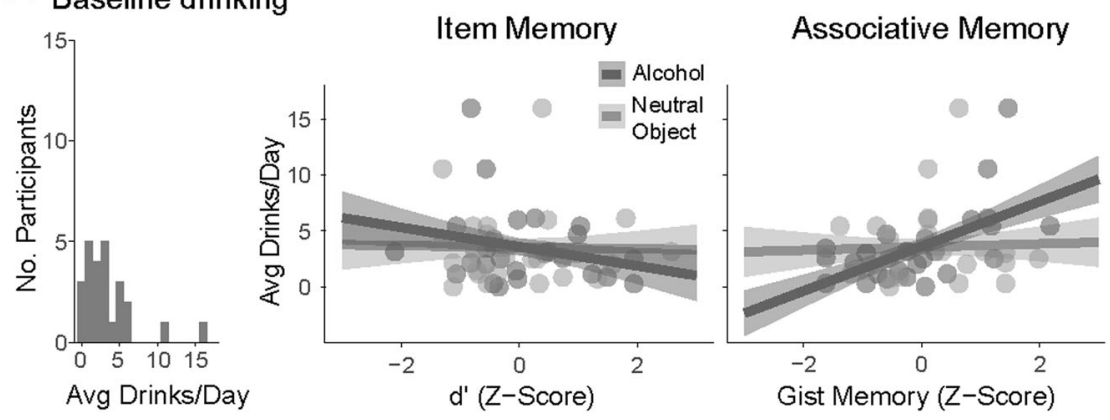

\section{B Prospective drinking}
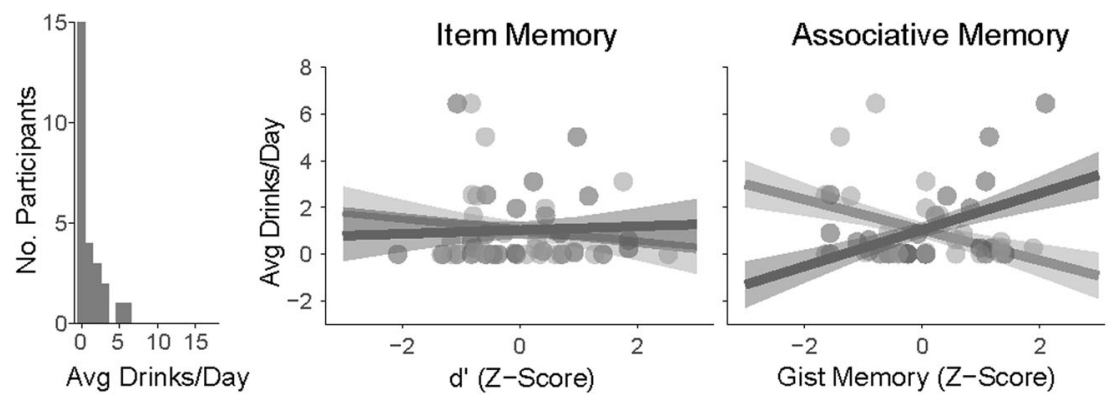

Fig. 4 Gist-level associative memory for alcohol predicts real-world drinking behavior. a Baseline drinking behavior (1 week prior to start of the study). b Subsequent drinking behavior (4 weeks following memory assessment during treatment). Distribution of average reported drinking behavior at each time point shown at the left for reference. Relationships between drinking behavior and normalized item memory (middle) and associative memory (right) are shown, separately for alcoholic beverages (red) and neutral objects (gray). In each plot, participants are represented by two dots: one for alcohol-related and one for neutral object-related memories. Error bars $=$ standard error of model coefficients.

more severe drinking within these patients (medium-/large- effect size). However, whereas detailed associative memory differentiated these groups, it was "gist"-level associative memory that predicted more severe drinking within AUD. These results were consistent across analyses: group differences in detailed associative memory were intensified with severe AUD, and the relationships between "gist"-level associative memory and drinking were replicated in two (uncorrelated) metrics of behavior. As several addiction models include nonlinear stages between recreational use and addiction (e.g., refs. $[66,67])$, it is not surprising that different aspects of associative memory would differentiate groups and linearly track escalating severity within AUD. In addition, our metric of escalating severity (more drinks per day) could mean something dramatically different for a moderate drinker ( 2 drinks/week) and a patient with AUD (38+ drinks/week). We previously showed that memories which distinguished groups (low vs. moderate lifetime stressor exposure) did not linearly predict incremental increases (more stressors) within each group, perhaps because a single stressor means something different in the context of low vs. moderate levels of lifetime stress [68]. This novel finding would be strengthened by replication in a preregistered sample.

These results highlight the importance of "gist"-level memory for alcohol-related associations for understanding drinking behavior. These memories could be interpreted as a broadened propensity to form associations with alcohol. Consistent with this idea, previous research showed that individuals who drink more associate a wider range of ambiguous cues with alcohol-related behaviors, and this broader activation of alcohol-related "memory associations" (although these were not tied to specific prior episodes) predicted increased drinking [38, 40, 69]. Similarly, heavier drinkers falsely recognized a broader set of alcohol-related expectancy words, particularly in an alcohol-related context [70]. The importance of this wide associative net for alcohol-related information emphasizes the need for treatments that address alcohol urges and use across a range of different contexts (i.e., in settings beyond the clinic) [2], and suggests that interventions that improve context discrimination could be beneficial. Another possibility is that less detailed memories for alcohol-related events would overshadow recall of specific negative drinking outcomes, making it difficult to learn the consequences of hazardous drinking (for further discussion of implications of overgeneralized autobiographical memory, see ref. [45]). From a neurobiological standpoint, gist-level emotional memory critically involves the amygdala [71], and gist-level associative memory has been linked to medial prefrontal cortex/hippocampal circuitry [72], regions in which function is dysregulated with chronic alcohol use [73, 74]. Notably, these memories were associated with both higher levels of baseline drinking and prospective drinking behavior while attempting to cut back. This suggests the potential utility for "gist"-level memories to understand both baseline severity of drinking behavior and the possibility of treatment success.

The influence of affective salience on item and associative memory in moderate drinkers is consistent with prior studies of emotional memory, with improved memory for items, but impaired memory for associates [26-28]. Intriguingly, patients with AUD did not show this pattern. This effect may be related to valence: although negative items have been linked to impaired associative memory, positive items were recently shown to benefit associative memory [75]. While patients with AUD had a trend toward rating more object/scene pairs as "happy" ( $p=0.08)$, this was not specific to alcohol-related trials (Cue $\times$ Group: $p=0.9$ ), making valence unlikely to explain differences in alcohol-related associative memory. These findings demonstrate that the affective salience attached to drug-related cues has distinct effects on memory in addicted populations, both compared with nonaddicted individuals and with typical effects of (non-drug-related) 
affective salience. It also raises the possibility that an affective process, like the salience ascribed to alcohol-related information by patients with AUD $[76,77]$, can explain both biases in memory and prospective drinking, although further work is needed to address this question. These results underscore the importance of measuring cognitive and affective processes in addicted populations for both drug-related and neutral information.

The limitations of the current experiment include demographic differences between the patients with AUD and moderate drinkers. It is important to note that this limitation is well documented in previous neurocognitive research, including AUD samples [21, 78]. While we cannot rule out the possibility that memory differences between groups could also be associated with these demographic differences, the study design (in addition to inclusion of these demographic features as covariates in all analyses) also minimized the potential influence of between-subject and group differences. By assessing memory for neutral objects as well as alcohol, we created a within-person individualized baseline that both accounts for global differences in memory performance due to participantlevel demographic features, and identifies memory biases specifically related to alcohol. Furthermore, the predictions of real-world drinking behavior were only conducted within patients with AUD and thus not subject to this limitation. We note that the relative advantage in memory for specific alcohol-related associations in AUD is even more striking, given that high age and low IQ are related to impairments in associative learning and memory $[79,80]$. Replication of these preliminary novel findings in demographically matched cohorts will facilitate stronger conclusions about the memory-related processes that contribute to AUD and support the design of optimized treatments that target these processes.

In conclusion, the current findings identify novel and meaningful biases in alcohol-related memory among patients with AUD relative to moderate drinkers. Critically, despite impairments in memory for items and associations with neutral objects, patients with AUD showed relatively enhanced memory for alcohol-related associations compared with moderate drinkers. Memory for alcohol-related associations also significantly and prospectively predicted heavier real-world drinking behavior among these patients. This work demonstrates the importance of exploring multiple dimensions of drug-related memories to both characterize and ultimately develop targeted treatments for individuals suffering from AUD.

\section{FUNDING AND DISCLOSURE}

This work was supported by National Institute of Health grants T32 DA022975 and CTSA KL2 TR001862 (EVG), R01 AA013892 (RS). The authors declare no competing interests.

\section{ACKNOWLEDGEMENTS}

The authors gratefully acknowledge Seungju Hwang, Chloe Larkin, Ryan Douglas, and Philip Himmelstein for assistance with recruitment and data collection.

\section{AUTHOR CONTRIBUTIONS}

EVG and RS conceived the work; EVG designed the work and supervised the data acquisition; EVG and NF analyzed and interpreted the data; EVG drafted the work; RS critically revised the work and approved it for publication.

\section{ADDITIONAL INFORMATION}

Supplementary Information accompanies this paper at (https://doi.org/10.1038/ s41386-020-0650-y)

Publisher's note Springer Nature remains neutral with regard to jurisdictional claims in published maps and institutional affiliations.

\section{REFERENCES}

1. Substance Abuse and Mental Health Services Administration (SAMHSA). NSDUH Series H-53. Rockville, MD: Center for Behavioral Health Statistics and Quality, Substance Abuse and Mental Health Services Administration; 2018.

2. Janak $\mathrm{PH}, \mathrm{Chaudhri} \mathrm{N}$. The potent effect of environmental context on relapse to alcohol-seeking after extinction. Open Addict J. 2010;3:76-87.

3. Crombag HS, Bossert JM, Koya E, Shaham Y. Review. Context-induced relapse to drug seeking: a review. Philos Trans R Soc Lond B Biol Sci. 2008;363: 3233-43.

4. Shaham Y, Shalev U, Lu L, de Wit H, Stewart J. The reinstatement model of drug relapse: history, methodology and major findings. Psychopharmacology. 2003;168:3-20.

5. Hyman SE, Malenka RC, Nestler EJ. Neural mechanisms of addiction: the role of reward-related learning and memory. Annu Rev Neurosci. 2006;29:565-98.

6. Torregrossa MM, Taylor JR. Neuroscience of learning and memory for addiction medicine: from habit formation to memory reconsolidation. Prog Brain Res. 2016;223:91-113.

7. Tiffany ST. A cognitive model of drug urges and drug-use behavior: role of automatic and nonautomatic processes. Psychol Rev. 1990;97:147-68.

8. Hyman SE. Addiction: a disease of learning and memory. Am J Psychiatry. 2005;162:1414-22.

9. Bornstein AM, Pickard H. "Chasing the first high": memory sampling in drug choice. Neuropsychopharmacology. 2020. https://doi.org/10.1038/s41386-0190594-2.

10. Heilig M, Augier E, Pfarr S, Sommer WH. Developing neuroscience-based treatments for alcohol addiction: a matter of choice? Transl Psychiatry. 2019;9:255.

11. White NM. Addictive drugs as reinforcers: multiple partial actions on memory systems. Addiction. 1996;91:921-49. discussion 51-65

12. Goldfarb EV, Sinha R. Drug-induced glucocorticoids and memory for substance use. Trends Neurosci. 2018;41:853-68.

13. Nadel L, Hardt O. Update on memory systems and processes. Neuropsychopharmacology 2011;36:251-73.

14. Cohen NJ, Poldrack RA, Eichenbaum H. Memory for items and memory for relations in the procedural/declarative memory framework. Memory 1997;5:131-78

15. Davachi L. Item, context and relational episodic encoding in humans. Curr Opin Neurobiol. 2006;16:693-700.

16. Henke K. A model for memory systems based on processing modes rather than consciousness. Nat Rev Neurosci. 2010;11:523-32.

17. Diana RA, Yonelinas AP, Ranganath $C$. Imaging recollection and familiarity in the medial temporal lobe: a three-component model. Trends Cogn Sci. 2007;11:379-86.

18. Acheson DT, Gresack JE, Risbrough VB. Hippocampal dysfunction effects on context memory: possible etiology for posttraumatic stress disorder. Neuropharmacology. 2012;62:674-85.

19. Bisby JA, Burgess N. Differential effects of negative emotion on memory for items and associations, and their relationship to intrusive imagery. Curr Opin Behav Sci. 2017;17:124-32.

20. Klein AA, Nelson LM, Anker JJ. Attention and recognition memory bias for alcohol-related stimuli among alcohol-dependent patients attending residential treatment. Addict Behav. 2013;38:1687-90.

21. Franken IHA, Rosso M, van Honk J. Selective memory for alcohol cues in alcoholics and its relation to craving. Cogn Ther Res. 2003;27:481-88.

22. Fridrici C, Driessen M, Wingenfeld K, Kremer G, Kissler J, Beblo T. Investigating biases of attention and memory for alcohol-related and negative words in alcohol-dependents with and without major depression after day-clinic treatment. Psychiatry Res. 2014;218:311-8.

23. Mellentin Al, Skot L, Nielsen B, Schippers GM, Nielsen AS, Stenager E, et al. Cue exposure therapy for the treatment of alcohol use disorders: a meta-analytic review. Clin Psychol Rev. 2017;57:195-207.

24. Das RK, Lawn W, Kamboj SK. Rewriting the valuation and salience of alcoholrelated stimuli via memory reconsolidation. Transl Psychiatry 2015;5:e645.

25. Valyear MD, Villaruel FR, Chaudhri N. Alcohol-seeking and relapse: a focus on incentive salience and contextual conditioning. Behav Process. 2017;141:26-32.

26. Bisby JA, Burgess N. Negative affect impairs associative memory but not item memory. Learn Mem. 2013;21:21-7.

27. Madan CR, Caplan JB, Lau CS, Fujiwara E. Emotional arousal does not enhance association-memory. J Mem Lang. 2012;66:695-716.

28. Touryan SR, Marian DE, Shimamura AP. Effect of negative emotional pictures on associative memory for peripheral information. Memory 2007:15:154-66.

29. Wiers RW, van Woerden N, Smulders FTY, de Jong PJ. Implicit and explicit alcohol-related cognitions in heavy and light drinkers. J Abnorm Psychol. 2002;111:648-58.

30. Carter BL, Tiffany ST. Meta-analysis of cue-reactivity in addiction research. Addiction. 1999;94:327-40. 
31. Wiers CE, Stelzel C, Park SQ, Gawron CK, Ludwig VU, Gutwinski S, et al. Neural correlates of alcohol-approach bias in alcohol addiction: the spirit is willing but the flesh is weak for spirits. Neuropsychopharmacology. 2014;39:688-97.

32. Reid LD, Hunter GA, Beaman CM, Hubbell CL. Toward understanding ethanol's capacity to be reinforcing: a conditioned place preference following injections of ethanol. Pharm Biochem Behav. 1985;22:483-7.

33. Gauvin DV, Holloway FA. Historical factors in the development of ETOHconditioned place preference. Alcohol. 1992;9:1-7.

34. Weafer J, Gallo DA, de Wit H. Acute effects of alcohol on encoding and consolidation of memory for emotional stimuli. J Stud Alcohol Drugs. 2016;77:86-94.

35. Goldfarb EV, Tompary A, Davachi L, Phelps EA. Acute stress throughout the memory cycle: diverging effects on associative and item memory. J Exp Psychol Gen. 2019;148:13-29.

36. Blaine SK, Sinha R. Alcohol, stress, and glucocorticoids: from risk to dependence and relapse in alcohol use disorders. Neuropharmacology. 2017;122:136-47.

37. Lee J, Im SJ, Lee SG, Stadlin A, Son JW, Shin CJ, et al. Volume of hippocampal subfields in patients with alcohol dependence. Psychiatry Res Neuroimaging. 2016;258:16-22.

38. Stacy AW. Memory association and ambiguous cues in models of alcohol and marijuana use. Exp Clin Psychopharmacol. 1995;3:183-94.

39. Stacy AW, Newcomb MD. Memory association and personality as predictors of alcohol use: mediation and moderator effects. Exp Clin Psychopharmacol. 1998;6:280-91.

40. Stacy AW. Memory activation and expectancy as prospective predictors of alcohol and marijuana use. J Abnorm Psychol. 1997;106:61-73.

41. Hogarth L, Duka T. Human nicotine conditioning requires explicit contingency knowledge: is addictive behaviour cognitively mediated? Psychopharmacology. 2006;184:553-66.

42. Hardy L, Mitchell C, Seabrooke T, Hogarth L. Drug cue reactivity involves hierarchical instrumental learning: evidence from a biconditional Pavlovian to instrumental transfer task. Psychopharmacology. 2017;234:1977-84.

43. Murty VP, FeldmanHall O, Hunter LE, Phelps EA, Davachi L. Episodic memories predict adaptive value-based decision-making. J Exp Psychol Gen. 2016;145:548-58.

44. Bornstein AM, Norman KA. Reinstated episodic context guides sampling-based decisions for reward. Nat Neurosci. 2017;20:997-1003.

45. Nandrino JL, Gandolphe MC, El Haj M. Autobiographical memory compromise in individuals with alcohol use disorders: towards implications for psychotherapy research. Drug Alcohol Depend. 2017;179:61-70.

46. Dunsmoor JE, Paz R. Fear generalization and anxiety: behavioral and neural mechanisms. Biol Psychiatry. 2015;78:336-43.

47. First MB, Williams JBW, Karg RS, Spitzer RL. Structured Clinical Interview for DSM5, Research Version. Arlington, VA: American Psychiatric Association; 2015.

48. Saunders JB, Aasland OG, Babor TF, de la Fuente JR, Grant M. Development of thE Alcohol Use Disorders Identification Test (AUDIT): WHO Collaborative Project on early detection of persons with harmful alcohol consumption-Il. Addiction. 1993;88:791-804.

49. Shipley WC. A self-administering scale for measuring intellectual impairment and deterioration. J Psychol. 1940;9:371-77.

50. Nowinski J, Baker S, Carroll K. Twelve step facilitation therapy manual: a clinical research guide for therapists treating individuals with alcohol abuse and dependence. Rockville, MD: National Institute on Alcohol Abuse and Alcoholism (NIAAA); 1992.

51. Dunsmoor JE, Martin A, LaBar KS. Role of conceptual knowledge in learning and retention of conditioned fear. Biol Psychol. 2012;89:300-5.

52. Xiao J, Hays J, Ehinger K, Oliva A, Torralba A. Xiao J, Hays J, Ehinger K, Oliva A, Torralba A. SUN Database: Large-scale Scene Recognition from Abbey to Zoo. IEEE Conference on Computer Vision and Pattern Recognition, 2010.

53. Miller WR, Del Boca FK. Measurement of drinking behavior using the Form 90 family of instruments. J Stud Alcohol Suppl. 1994;12:112-8.

54. Sobell LC, Sobell MB. Timeline Follow-Back. In: Litten RZ, Allen JP, eds. Measuring Alcohol Consumption. Totowa, NJ: Humana Press; 1992.

55. Wemm SE, Larkin C, Hermes G, Tennen H, Sinha R. A day-by-day prospective analysis of stress, craving and risk of next day alcohol intake during Alcohol Use Disorder treatment. Drug Alcohol Depend. 2019;204:107569.
56. $\mathrm{R}$ Core Team. R: A language and environment for statistical computing. $\mathrm{R}$ Foundation for Statistical Computing, Vienna, Austria. https://www.R-project.org/. 2017.

57. Pinheiro J, Bates D, DebRoy S, Sarkar D, R Core Team. nlme: linear and nonlinear mixed effects models. https://CRAN.R-project.org/package=nlme. 2020.

58. Tippey KG, Longnecker MT. An ad hoc method for computing pseudo effect size for mixed models. San Antonio, TX: SAS Conference Proceedings; 2016.

59. Cohen J. Statistical power analysis for the behavioral sciences. 2nd ed. Hillsdale, NJ: Lawrence Erlbaum Associates; 1988.

60. Bates ME, Bowden SC, Barry D. Neurocognitive impairment associated with alcohol use disorders: implications for treatment. Exp Clin Psychopharmacol. 2002;10:193-212.

61. Pitel AL, Beaunieux $H$, Witkowski T, Vabret F, Guillery-Girard B, Quinette $P$, et al Genuine episodic memory deficits and executive dysfunctions in alcoholic subjects early in abstinence. Alcohol Clin Exp Res. 2007;31:1169-78.

62. Kutlu MG, Gould TJ. Effects of drugs of abuse on hippocampal plasticity and hippocampus-dependent learning and memory: contributions to development and maintenance of addiction. Learn Mem. 2016;23:515-33.

63. Le Berre AP, Fama R, Sullivan EV. Executive functions, memory, and social cognitive deficits and recovery in chronic alcoholism: a critical review to inform future research. Alcohol Clin Exp Res. 2017;41:1432-43.

64. Wimmer GE, Shohamy D. Preference by association: how memory mechanisms in the hippocampus bias decisions. Science 2012;338:270-3.

65. Bornstein AM, Khaw MW, Shohamy D, Daw ND. Reminders of past choices bias decisions for reward in humans. Nat Commun 2017;8:15958.

66. Piazza PV, Deroche-Gamonet V. A multistep general theory of transition to addiction. Psychopharmacology. 2013;229:387-413.

67. Koob GF, Volkow ND. Neurobiology of addiction: a neurocircuitry analysis. Lancet Psychiatry. 2016;3:760-73.

68. Goldfarb EV, Shields GS, Daw ND, Slavich GM, Phelps EA. Low lifetime stress exposure is associated with reduced stimulus-response memory. Learn Mem. 2017;24:162-68.

69. Woud ML, Becker ES, Rinck M, Salemink E. The relationship between drinking motives and alcohol-related interpretation biases. J Behav Ther Exp Psychiatry. 2015;47:102-10.

70. Reich RR, Goldman MS, Noll JA. Using the false memory paradigm to test two key elements of alcohol expectancy theory. Exp Clin Psychopharmacol. 2004;12: 102-10.

71. Adolphs R, Tranel D, Buchanan TW. Amygdala damage impairs emotional memory for gist but not details of complex stimuli. Nat Neurosci. 2005;8: 512-8.

72. Berkers R, Klumpers F, Fernandez G. Medial prefrontal-hippocampal connectivity during emotional memory encoding predicts individual differences in the loss of associative memory specificity. Neurobiol Learn Mem. 2016;134: 44-54.

73. Gilpin NW, Herman MA, Roberto $M$. The central amygdala as an integrative hub for anxiety and alcohol use disorders. Biol Psychiatry. 2015;77:859-69.

74. Seo D, Lacadie CM, Tuit K, Hong Kl, Constable RT, Sinha R. Disrupted ventromedial prefrontal function, alcohol craving, and subsequent relapse risk. JAMA Psychiatry. 2013;70:727-39.

75. Madan CR, Scott SME, Kensinger EA. Positive emotion enhances associationmemory. Emotion. 2019;19:733-40.

76. Robinson $\mathrm{TE}$, Berridge $\mathrm{KC}$. The neural basis of drug craving: an incentivesensitization theory of addiction. Brain Res Brain Res Rev. 1993;18:247-91.

77. Cofresi RU, Bartholow BD, Piasecki TM. Evidence for incentive salience sensitization as a pathway to alcohol use disorder. Neurosci Biobehav Rev. 2019;107:897-926.

78. Squeglia LM, Gray KM. Alcohol and drug use and the developing brain. Curr Psychiatry Rep. 2016;18:46.

79. Kaufman SB, DeYoung CG, Gray JR, Brown J, Mackintosh N. Associative learning predicts intelligence above and beyond working memory and processing speed. Intelligence. 2009;37:374-82.

80. Naveh-Benjamin M. Adult age differences in memory performance: tests of an associative deficit hypothesis. J Exp Psychol Learn Mem Cogn. 2000;26:1170-87. 